\title{
VECTOR MESON PRODUCTION ABOVE THE RESONANCE REGION
}

\author{
MARCO BATTAGLIERI \\ On behalf of the CLAS Collaboration \\ Istituto Nazioneale di Fisica Nucleare Via Dodecaneso 33, 16146 Genov, Italy
}

\begin{abstract}
The total and differential cross section for exclusive vector meson electro- and photoproduction above the resonance region $(2<W<3 \mathrm{GeV})$ were measured at Jefferson Laboratory covering a wide kinematic range $\left(0<Q^{2}<5 \mathrm{GeV}^{2} / c^{2}\right.$ and $0<-t<5$ $\mathrm{GeV}^{2}$ ). These measurements provide a unique tool to investigate the transition between soft and hard physics. Our data indicates that this regime can be successfully and consistently described by using the so called effective partonic degrees of freedom.
\end{abstract}

Keywords: Vector mesons; Regge trajectories; large momentum transfer.

\section{Introduction}

One of the key issues in nuclear physics is how to link the low energy and low momentum transfer regime to the high energy and large momentum transfer domain. While the former can be described by constituent quarks or phenomenological models using effective degrees of freedom, the latter uses true QCD fields in the perturbative approach. The specific kinematic region of large momentum transfer but moderate energy can help to understand the link between the the soft and the hard physics. Large momentum transfer corresponds to a small impact parameter $(b \sim 1 / \sqrt{-t})$ : at short distances where QCD asymptotic freedom dominates, one expects the complexity of hadron scattering to be reduced to a simpler picture based on elementary partons. Factorization is the key issue that allows one to mix perturbative QCD with low energy regime. There are two possible frameworks to consistently describe the low energy physics using the high energy language: the handbag factorization ${ }^{1,2}$ where only one active parton has a hard interaction with the incoming photon leaving the soft physics parametrized trough the formalism of the Generalized Parton Distributions; the leading twist factorization ${ }^{3}$ where all partons are involved in the scattering and the soft physics is encoded in the nonperturbative wave-function (the Distribution Amplitudes). In the second scheme the dimensional counting rules a 5 were derived and extensively applied to the phe-

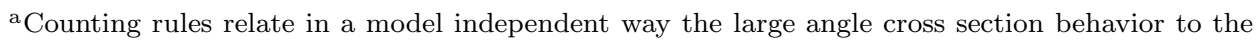
number of constituent partons involved in the scattering. In this sense the behavior predicted by counting rules is an indication of hard scattering. 
nomenology of large transverse momentum reactions in hadron scattering ${ }^{4}$ in a similar kinematic region $\left(s \sim 5-10 \mathrm{GeV}^{2}\right.$ and $\left.t \sim 3-6 \mathrm{GeV}^{2}\right)$. We will see that depending on the reaction one or the other approach is better suited to describe our data.

The electromagnetic interaction is weak enough to be treated perturbatively and exclusive processes involving one or more photons are, at leading twist, insensitive to long-distance physics revealing a precocious hard behavior. ${ }^{6}$ In particular, according to the vector dominance hypothesis, photoproduction shows common features with hadronic scattering; if the photon energy is high enough $\left(E_{\gamma}>2 \mathrm{GeV}\right)$ the photon can fluctuate into a $(q \bar{q})$ state that propagates and interacts with the target nucleon. This picture is confirmed by comparing the hadronic elastic total cross sections to the photo-absorption: ${ }^{7}$ all of them are simply explained by the exchange of the Pomeron and other few Regge trajectories $\left(f_{2}\right.$ and $\pi$ ). The same universal behavior is also evident when the differential cross sections are compared. ${ }^{8}$

It is well known that Regge theory is an economic and powerful tool to describe both exclusive and inclusive soft hadronic processes but it is a phenomenological approach not dealing with quarks and gluons. In the last years several attempts have been made to evaluate the photo production cross sections at leading twist within $\mathrm{pQCD}{ }^{9}$ assuming the factorization of the hard scattering amplitudes (pQCD calculable) and the distribution amplitudes. Only a qualitative agreement between calculations and experimental data was obtained. Soft physics corrections (e.g quark-diquark models ${ }^{10}$ ) improve the agreement but still fail to achieve a complete description in this regime within the hard-scattering approach.

An alternative approach 11,12,13 describes the transition domain by using effective partonic degrees of freedom. It uses the Regge phenomenology but interprets the Regge quanta in terms of QCD fields: the Pomeron exchange in terms of two-gluon exchange and the Regge trajectories in terms of quark exchange. Nonperturbative effects are taken into account by using dressed gluons, constituent quark propagators, and parton correlations in the nucleon wave function. It is therefore easy to relate experimental observables (total and differential cross section, polarization etc.) to fundamental and truly non-perturbative quantities like propagators or distribution amplitudes, which can be calculated using lattice-QCD. In this approach the link between the forward (t-exchange) and the backward (u-exchange) peaks is obtained by using the saturated Regge trajectories, i.e. $t$-independent at large momentum transfer. ${ }^{14,15}$ Saturated trajectories lead to the asymptotic quark counting rules. ${ }^{5}$ This approach was successfully adopted to explain the large momentum transfer hadron - hadron interactions as well as several photon induced reactions. The simultaneous description of many channels has been used to fix all free parameters of the model and test the validity of this picture.

\section{Vector meson production}

Vector mesons $(\rho, \omega$ and $\phi$ ) have the same quantum numbers as the photon $\left(J^{P C}=1^{--}\right)$but different flavor composition. For all of them, the differential 
cross section shows two well defined dynamical regimes depending on the momentum transfer $-t$. The low $-t$ region $\left(-t<1 \mathrm{GeV}^{2}\right)$ shows a diffractive behavior interpreted in the frame of the Vector Meson Dominance model as the elastic scattering of vector mesons off the proton target. At high $-t\left(-t>1 \mathrm{GeV}^{2}\right)$, where the cross section should be sensitive to the microscopic details of the interaction, the underlying physics can be described using parton degrees of freedom. The small impact parameter $(\approx 1 / \sqrt{-t})$ resolves the two-gluon (two-quark) exchange mechanisms by preventing the the constituent gluons (quarks) of the exchange to interact and form a Pomeron (Reggeon). Moreover, small transverse sizes select configurations where each gluon couples to different quarks both in the vector meson and the nucleon, giving access to the correlation function in the proton. ${ }^{12}$ Due to the dominant $s \bar{s}$ component of the $\phi$, and to the extent that the strangeness of the proton is small, the $\phi$ photoproduction at large $-t$ is a good tool to resolve the Pomeron into its simplest 2-gluon component. In the $\rho$ and $\omega$ cases, their light quark composition also allows valence quarks to be exchanged between the baryon and the meson states. ${ }^{12}$ The comparison between the three channels puts stringent constraints on our understanding of reaction mechanisms.

The virtual photon allows the complementary extention of exploration of the nucleon and its components. In fact, while the dynamics can be fixed at the real photon point, increasing the photon virtuality $\left(Q^{2}>0\right)$ the lifetime decreases. The coupling between the photon and the nucleus (or its constituents) becomes more and more point-like allowing us to probe shorter distances. Again, using the $Q^{2}$ as the tuning parameter, the hope we have is to reveal the interaction mechanisms and the structure of the exchanged quanta in terms of fundamental QCD degrees of freedom.

\section{Experimental setup and data analysis}

The vector meson data were obtained with the CEBAF Large Acceptance Spectrometer (CLAS) in the Hall B of the Thomas Jefferson National Accelerator Facility. CLAS is a nearly $4 \pi$ spectrometer based on a toroidal magnetic field generated by six superconducting coils. Three drift chambers regions allowed tracking of charged particles; time of flight scintillators were used for hadron identification. The experiment was performed using both the $6 \mathrm{GeV}$ primary electron beam (in the kinematic range $W>2 \mathrm{GeV}$ and $Q^{2}$ up to $6 \mathrm{GeV}^{2}$ ) and a secondary high intensity $\left(6 \times 10^{6} \gamma / s\right)$ bremsstrahlung photon beam, tagged in the $E_{\gamma}$ range between 3 to 4 $\mathrm{GeV}$.

The exclusive production of $\phi, \rho$ and $\omega$ were uniquely identified by direct measurements of the decay products in combination with appropriate missing-mass constraints. The reaction $\gamma_{r / v} p \rightarrow p \phi$ was reconstructed by measuring the proton and $K^{+}$and determining the $K^{-}$momentum using energy and momentum conservation. The number of $\phi$ mesons in each $t$-bin was determined by subtracting the non-resonant contribution under the $\phi$ peak in the invariant $\left(K^{+} K^{-}\right)$mass spec- 


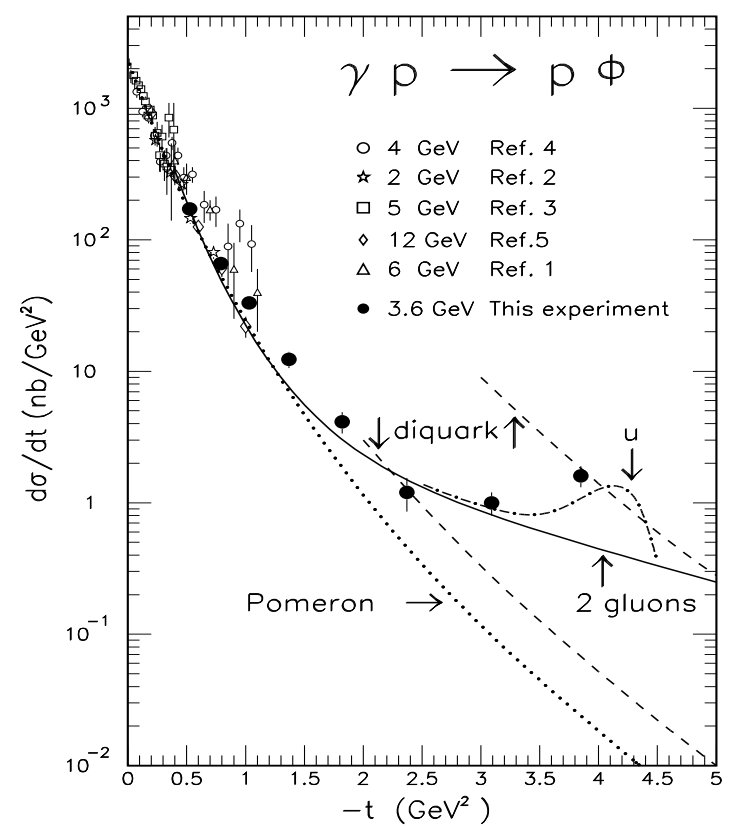

Fig. 1. The differential cross section of the reaction $\gamma p \rightarrow p \phi$. See text for explanation.

trum in different ways (fitting the shape of the data and using a simple model). The reaction $\gamma_{r / v} p \rightarrow p \rho^{0}$ with $\rho^{0} \rightarrow \pi^{+} \pi^{-}$was measured by detecting all possible final-state with at least two hadrons and using the missing mass technique when necessary. For each $\left(E_{\gamma},-t\right)$ bin, a simultaneous fit of both the invariant mass $\left(p \pi^{+}\right)$and $\left(\pi^{+} \pi^{-}\right)$was performed in order to separate the $\rho$ production from the other channels $\left(\Delta^{0} \pi^{+}, \Delta^{++} \pi^{-}\right.$, phase space, $\left.f_{2}(1270)\right)$ contributing to the $p \pi^{+} \pi^{-}$ final state. The reaction $\gamma_{r / v} p \rightarrow p \omega$ was detected via the main decay channel $\omega \rightarrow \pi^{+} \pi^{-} \pi^{0}$ using a combination of the procedures described above.

\section{Results}

The measured $d \sigma / d t$ for elastic $\phi$ photoproduction on the proton, ${ }^{16}$ is shown in Fig. 1; at low momentum transfer the two-gluon exchange model of J.M. Laget and R. Mendez-Galain ${ }^{12,11}$ (solid line) coincides with the well known diffractive behavior (dotted line). For $-t>2 \mathrm{GeV}^{2}$ the data rule out the diffractive Pomeron exchange and strongly favor its two-gluon realization. In the calculation the gluons can couple to any quarks in the baryon. Quark correlations in the proton are taken into account assuming the simplest form of its wave function with three valence quarks equally sharing the proton longitudinal momentum. In the same plot two perturbative QCD calculations are also shown (dashed lines). Neither is able to describe the data demonstrating that the asymptotic regime is not yet reached. The $\rho^{17}$ and the $\omega^{18}$ differential cross sections are shown in Fig. 2. The parameters of 
the two-gluon exchange mechanism are fixed from the analysis of the $\phi$. The low momentum transfer region is well reproduced when the two Regge trajectories $\left(f_{2}\right.$ and $\sigma$ for the $\rho$ and $f_{2}$ and $\pi$ for the $\omega$ ) are included while the model underestimates the large $-t$ region, indicating that other reaction mechanisms are needed. The good agreement with data is obtained (solid line) when quark-exchange processes are added through the saturated trajectory technique. The same model using nonsaturated trajectories is also shown (dot-dashed line). The power law $s^{-C}$ fit to $d \sigma / d t$ at $90^{\circ}$ in the center of mass was performed using CLAS and world-data. The fit yields $C=7.9 \pm 0.3\left(\chi^{2}=0.6\right)$ for the $\rho$ and $C=7.2 \pm 0.7\left(\chi^{2}=0.4\right)$ for the $\omega$ channel. It is the first time that such a power law behavior has been observed in the $\omega$ channel, although it has been seen in other exclusive reactions. The pointlike interaction and the hadronic component of the photon have a $s^{-7}$ and $s^{-8}$ power-law behavior by dimensional counting. ${ }^{5}$ Note that also the saturated $\sigma$ and $\pi$ Regge trajectories behave like $s^{-8}$. In addition to the differential cross section at fixed energy, the $s$-dependence is a strong hint of the presence of hard quark interchange mechanisms in addition to the two-gluon exchange.

As far as the electroproduction is concerned, our data show that the $\rho^{19}$ and the $\omega$ have a very different behavior. In the kinematic range to which we have access, the longitudinal part start to dominate the $\rho$ cross section allowing an interpretation based on the GPD's formalism b. The transverse cross section can be described by the same Regge-based model with a $Q^{2}$ monopole form-factor. In the case of the $\omega$ electroproduction the pion-exchange dominates even at large $Q^{2}$ (our data reach $\mathrm{Q}^{2} \sim 5 \mathrm{GeV}^{2}$,) violating the $\mathrm{SCHC}$ and then not allowing a clear separation between the longitudinal and the transverse part. In this case the factorization is not valid and, at least in our kinematic domain, a GPD based interpretation is not possible. For this channel too, the Regge approach has been extended ${ }^{20}$ to take into account the $Q^{2}$ dependence. Two kinematic domains can be identified depending on the momentum transfe: in the low $-t$ region, a hadronic form factor (the exchanged pion form factor), well describes the exponential behavior of the differential cross section $d \sigma / d t$; at large $-t, d \sigma / d t$ flattens-out, even at large $Q^{2}$ revealing the coupling of the virtual photon to point-like objects.

\section{Conclusion}

Vector meson photo- and electro-production above the resonance region is a unique tool to study and understand the quark-gluon structure of hadronic matter. At Jefferson Lab a set of dedicated experiments has been completed with both real and virtual photons covering a wide kinematic range: $2<W<3 \mathrm{GeV}, 0<Q^{2}<$ $5 \mathrm{GeV}^{2} / \mathrm{c}^{2}$ and $-t$ up to $5 \mathrm{GeV}^{2}$. The production of $\phi, \rho$ and $\omega$ mesons were analyzed in the same framework simultaneously. At large momentum transfer the $\phi$ data

\footnotetext{
b It has been proved that the handbag diagram-based factorization is only valid for longitudinal photons and therefore, assuming that the photon helicity is transfered to the VM, only the longitudinal part of the cross section can be interpreted within the GPD formalism.
} 

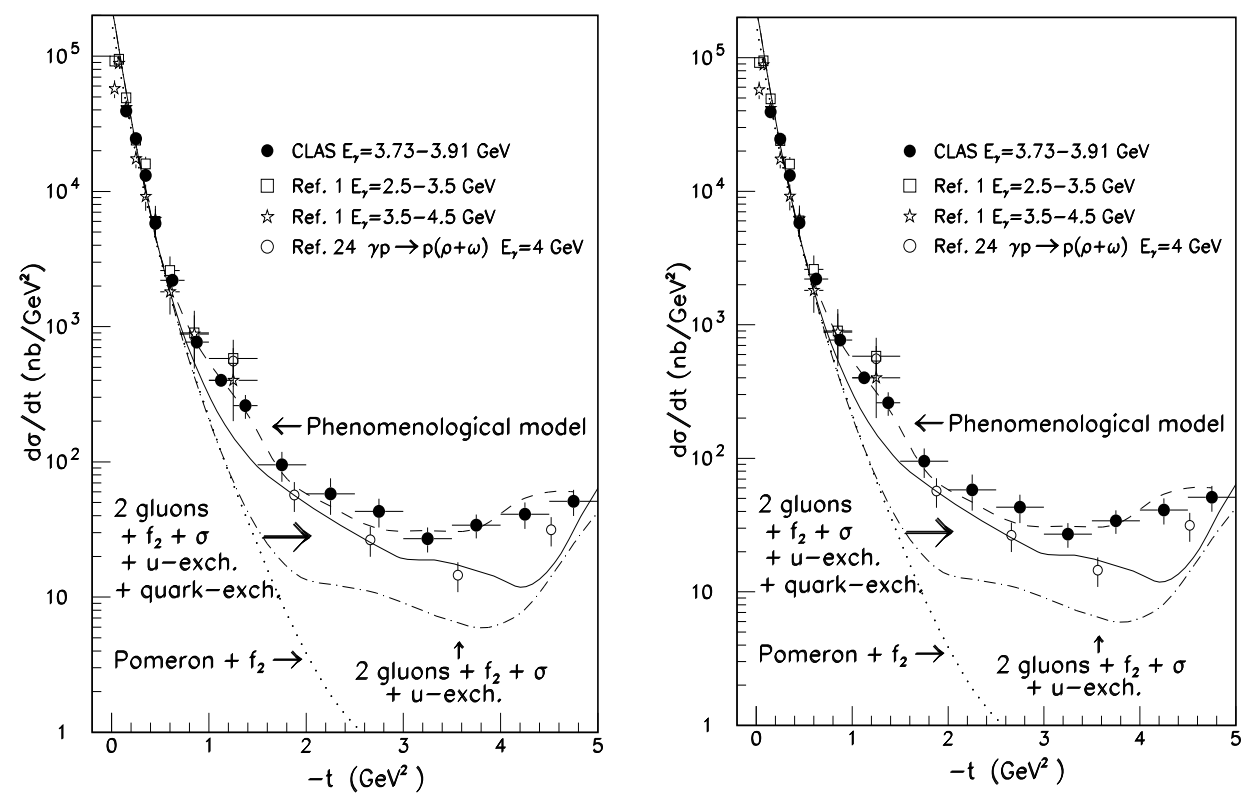

Fig. 2. The differential cross section of reactions $\gamma_{r / v} p \rightarrow p \rho$ and $\gamma p \rightarrow p \omega$. See text for explanation.

support a model where the Pomeron is resolved into its simplest component, two gluons, which may couple to any quark in the proton and in the vector meson. The $\rho$ and the $\omega$ data indicate that other processes, such as quark interchange, are required to fully describe these channels. The two scales $t$ and $Q^{2}$ allows the exploration of the space-time structure of the hard scattering process: at low $Q^{2}$ (and in the realphoton limit) increasing $t$ allows to directly look at the dynamics of the exchanged objects. At large $Q^{2}$, the coupling becomes more and more point-like, probing the short distance structure of the coupling vertexes. In conclusion, a consistent picture is emerging from $\phi, \rho$ and $\omega$ electro and photoproduction showing that this kinetic region can be described as a non perturbative partonic regime, where microscopic degrees of freedom (gluons and quarks) are combined with a low-energy picture of hadrons (constituent quarks, dressed gluons).

\section{References}

1. X. Ji, Phys. Rev. D 55, 7114 (1997).

2. A. Radyushkin, Phys. Rev. D 56, 5524 (1997).

3. G. Lepage and S.J. Brodsky, Phys. Rev. D 22, 2157 (1980).

4. C. White et al., Phys. Rev. D 49, 58 (1994).

5. S.J. Brodsky and G.R. Farrar, Phys. Rev. Lett. 31, 1153 (1973).

6. G.R. Farrar et al., Phys. Rev. Lett. 62, 2229 (1989).

7. A. Donnachie, P.V. Landshoff, Phys. Lett.B296, 227 (1992).

8. R.L. Anderson et al., Phys. Rev. D 14, 679 (1976).

9. G.R. Farrar et al., et al., Nucl. Phys. B349, 655 (1991). 
10. P. Kroll et al., Phys. Rev. D 55, 4315 (1997).

11. J.M. Laget and R. Mendez-Galain, Nucl. Phys. A581, 397 (1995).

12. J.M. Laget, Phys. Lett. B489, 313 (2000).

13. F. Cano and J.M. Laget, Phys. Rev. D 65, 074022 (2002).

14. M.N. Sergeenko, Z. Phys. C 64, 315 (1994).

15. M. Brisudova et al., Phys. Rev. D 61, 054013 (2000).

16. E. Anciant et al., Phys. Rev. Lett. 85, 4682 (2000).

17. M. Battaglieri et al., Phys. Rev. Lett. 87, 172002 (2001).

18. M. Battaglieri et al., Phys. Rev. Lett. 90, 022002 (2003).

19. C. Hadjidakis et al., preprint hep-ex/0408005

20. J.M. Laget, preprint hep-ph/0406153 